\section{Europhysics Notes}

\section{- High-T $T_{c}$ Surprises}

High-temperature superconductivity recaptured the public's attention in late December (and stimulated specialists in the field) with anouncements by CNRS groups in France. Tholence et al. based in Grenoble reported in Phys. Lett. A (3 Jan. 1994) possible evidence from electrical resistivity measurements of traces of a superconducting phase with a $T_{c}$ of around $250 \mathrm{~K}$ in a $\mathrm{HgBaCaCuO}$ compound produced under pressure to facilitate the formation reactions. The anomalies disappeared after thermal cycling so reproducibility seems to be a problem. As the authors themselves remark, "more experiments are needed to confirm the existance of a phase superconducting at around $250 \mathrm{~K}$ ". More recent but unpublished magnetic susceptibility work suggests that the observed anomalies are not artifacts easily explained by other effects, but in a fax to a conference in the USA at the end of January the group indicated that "the data had not yet been reproduced in a systematic way". The other announcement concerned a sequentially deposited cuprate film belonging to the $\mathrm{BiSrCaCuO}$ family with eight adjacent $\mathrm{CuO}_{2}$ layers. Lagues et al. from Paris reported in Nature 262 (1993) 1850 that $i-V$ curves displayed a transition to low resistivity at temperatures below about $270-280 \mathrm{~K}$. Something seems to be happening as a US group at Varian (which is probably the best placed to reproduce the results) appears to have seen a similar, although much weaker, effect. However, confirmation that it is related to superconductivity remains lacking.

\section{- Three Detectors by 2000}

The 1st Edoardo Amaldi Conference on Gravitational Wave Experiments (14-17 June 1994; Villa Tuscolana, Frascati) organized by the INFN will honour Amaldi for his rôle in promoting gravitational wave research (contact L. Catena; fax.: +39-6-202 35 07; catena @ roma2.infn.it). The event essentially follows on from a similar meeting last year [see EN 24 (1994) 112] that aimed to promote coordination in a field which received a big boost in December when the INFN decided to go ahead with the large, French-Italian VIRGO laser interferometer to be constructed in six years near Pisa. The lack of capital investment funds means that there is no formal

A map showing the location near Pisa of VIRGO's two, $3 \mathrm{~km}$ long interferometer arms.

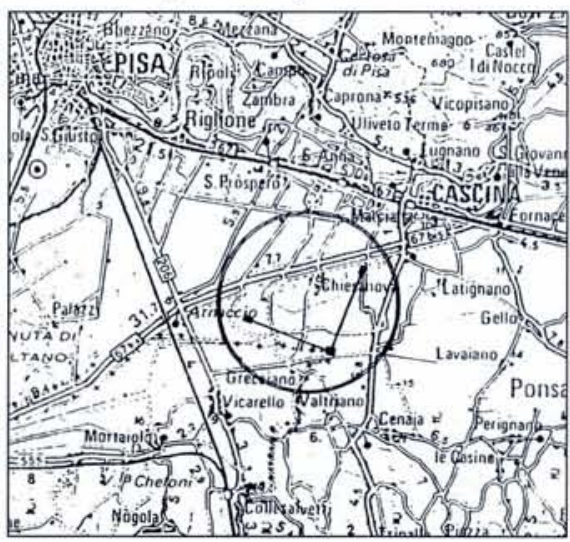

\section{DEPARTMENT of PHYSICS \& ASTRONOMY} TAIT CHAIR OF MATHEMATICAL PHYSICS

The University invites applications for appointment to the Tait Chair of Mathematical Physics, with effect from Ist October, 1994, or as soon as possible thereafter. The post has fallen vacant through the appointment of Professor D J Wallace, FRS, as Vice-Chancellor of Loughborough University of Technology.

The University seeks an established researcher who has made significant contributions to any branch of theoretical physics. Research areas within the Department include: Applied Optics, Astronomy (linked to the SERC Royal Observatory Edinburgh), Computational Physics, Condensed Matter, Fluids, Nuclear and Particle Physics, and theoretical Physics. The research interests of the Theoretical Physics Group, headed by Professor P W Higgs, FRS, are predominantly in elementary particle physics. The Group plays a leading role in the UKQCD project and has strong links with the Edinburgh Parallel Computing Centre.

Please quote REF: EN 940031. Further particulars may be obtained from: THE SECRETARY TO THE UNIVERSITY, THE UNIVERSITY OF EDINBURGH, I ROXBURGH STREET, EDINBURGH EH8 9TB, Fax: 031-650 6509

with whom applications ( 14 copies). including names and addresses of three referees, should be lodged. Overseas applicants need send only one copy of their application. Applications by fax should be confirmed by mail. Closing date: 2nd May, 1994.

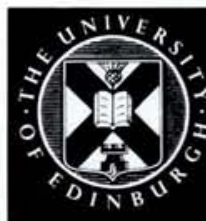

\section{2nd European Seminar in Telemedicine 18-22 April 1994 - Anavissos, Athens, Greece}

Review talks: Framework; Information in health care; Image storage, retreival, processing \& analysis; Communications \& networks; Medical evaluation of digital images Workshops: FEST case studies/Question set; Image processing/DIAS software; Medical records/Health 01 software; Telemedicine user interface \& DARE image bank (Essen);

Workstation evaluation \& ROC analysis

Presentations: Greek Telemedicine Programme; EU programme; Economic aspects

The school addresses mainly young physicians who want to acquire a good knowledge of telemedicine. A few engineers and administrators will also be admitted.

No attendance fee - Limited number of fellowships available - Limited to 30

Deadlines: Applications - 15 March 1994; for financial support - 1 March 1994

Contact: D. Sotiriou, Dept. of Medical Physics, School of Medicine, Athens Univ., Mikras Assias 75, Goudi, GR-115 27 Athens. Email: sotiriou@alpha.telemed.adriane-t.gr; tel.: +30-1-7793 273/778819; fax +30-1-7793 273/778819.

\section{Postdoctoral Positions and Guest Professorships at the Center for Atomic-scale Materials Physics (CAMP)}

At the Center for Atomic-scale Materials Physics (CAMP), which is a newly established research institute under the Danish National Research Foundation, a number of postdoctoral fellowships and guest professorships will be available starting in 1994 or later. Applications are invited from both experimentalists and theorists.

The experimental research is centered at the Institute of Physics and Astronomy, Aarhus University, Denmark. The main topics of interest are metal-on-metal growth, atom-scale material properties and chemical reactions on surfaces studied by scanning tunneling microscopy and a range of other surface-sensitive analytical techniques.

The theoretical research is centered at the Physics Department of the Technical University of Denmark in Lyngby, just outside Copenhagen. The main topics of interest are "ab initio" electronic structure and total-energy calculations for solids, clusters and surfaces, approximate total-energy methods and atomistic simulations of materials properties and chemical reactions at surfaces.

Applications should include a curriculum vitae, a statement of research interests, a list of publications, and for the postdoc positions names of three referees.

For further information, please contact the Director, Professor Jens K. Nørskov, CAMP Physics Department, Building 307, Technical University of Denmark, DK-2800 Lyngy, phone: +45 45931222 - 3175, fax: +45 459323 99, e-mail: norskov@fysik.dth.dk, or the Vice-Director, Professor Flemming Besenbacher, CAMP, Institute of Physics and Astronomy, Aarhus University, DK-8000 Aarhus C, phone: +45894236 04, fax: +45 861207 40, e-mail: fbe@dfi.aau.dk.

Applications should be sent as soon as possible to the Director of CAMP at the above address. 
collaboration between VIRGO and UK and German groups based on the GEO team that proposed an antenna a few years ago. However, Karsten Danzmann from Hannover University and the Max Planck Institute has indicated that German efforts are being consolidated in Hannover and a $600 \mathrm{~m}$ prototype interferometer located next to the Laser Centre is planned. It would be used for research and to test next-generation concepts. The two large LIGO detectors under construction in the US received a positive review in a recent appraisal, but completion in 1996-97 now seems unrealistc owing to budget reductions.

\section{- First Light at ELETTRA}

Commissioning of Trieste's ELETTRA 2 GeV ultra-high brilliance synchrotron radiation source began last October and a beam was stored after only one week (the design current of $400 \mathrm{~mA}$ at $1.1 \mathrm{GeV}$ was reached by mid-December). Two undulator sections have been installed and light from one was first seen on 7 November. As conditioning the $1.5 \mathrm{GeV}$ linac used for preacceleration is taking longer than expected (1.25 GeV has been reached), a procedure has been set up for ramping to $1.5 \mathrm{GeV}$. It is planned to start user operations at the first two beam lines in early July, with a further two lines becoming available in September. ELETTRA is open to external users and a document defining the users policy will be released shortly.

\section{- Hubble Repair a Success}

NASA released Hubble Space Telescope images in mid-January showing that the corrective optics used in the new Wide Field and Planetary Camera installed during December's Shuttle repair mission compensates fully for the optical aberation of Hubble's primary mirror. Moreover, the COSTAR device installed to feed a corrected image to ESA's Faint Object Camera allows the FOC to focus better than was originally intended. COSTAR also feeds light to two spectrographs, one of which is being aligned while the other is under test (the littleused High-Speed Photometer was removed). The Space Telescope Science Institute in Baltimore, USA, which manages the overall scientific utilization of the HST is not expected to experience much change as a result of the repair mission's success. A general, NASA-driven, belt tightening ( $10 \%$ staff reduction compared to pre-repair days) will have little impact as the operation has become more routine with fewer problems to circumvent. A higher throughput is possible without HSP aboard and with exposure times and loss of guide stars reduced, but this will be compensated for by looking at fainter objects. Europe's astronomers have done well in competing for viewing time as they were being assigned $22 \%$ of the time before the repair whereas a 1990 agreement calls for $15 \%$ on the basis of ESA's contributions.

\section{- National Debate Launched}

The national debate on the "main directions" for science in France launched last year by the Minister for research and higher education [EN 24 (1993) 188] has, in a widely circulated "pre-report", chosen the themes and offered some ideas which go to the heart of how French research functions. The themes focus heavily, but not exclusively, on national issues, mainly the structure of research (the notion that the

\section{PC'94: 6th Joint EPS-APS International Conference on Physics Computation \\ Lugano, Switzerland}

22-26 August 1994

\section{Organized by the Swiss Scientific Computing Centre}

PC'94 aims to bring together scientists and engineers interested in innovative approaches in computational physics. Special emphasis will be given to algorithims for, and the implentation of, high-performance computing.

PC '94 will give a detailed overview of the latest results of research and development in computational methodolgy by both academia and industry. There will be invited and contributed papers, poster sessions, tutorials, and commercial exhibits. Proceedings will be available at the conference.

\section{For further information, contact.}

PC'94, c/o CSCS, CH-6928 Manno, Switzerland

Tel.: +41-91-508211; Fax: +41-91-506711; E-mail: pc94@ cscs.ch
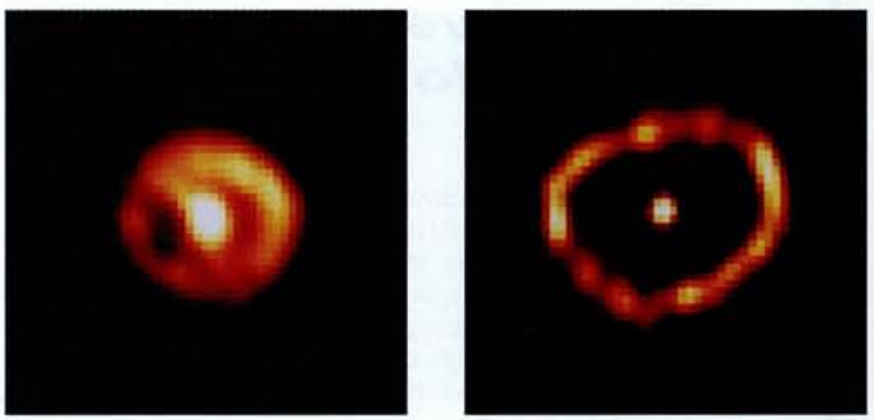

The May 1993 FOC raw image (on the left) does not resolve the edge of a ballooning bubble of hot gas expelled from a white dwarf star (Nova Cygni 1992) following a thermonuclear explosion on the surface because spherical aberation scattered light from the star. The raw image (on the right) taken using COSTAR's corrective optics reveals the ring-like structure of the expanding shell ot gas, thereby illustrating the greatly improved sensitivity and resolution.

administration can itself decide not to support a given piece of work is suggested), increasing student numbers in the universities (it is proposed to use research staff with permanent positions to teach), and the separation between research and industry (closer contacts between research organizations and medium and small industry are sought). One of seven colloquia will consider the international context (Lille; 17 March) and the final report (at the end of April) will be followed by a full debate in Paris.

\section{- Proposed Source Meets Some Resistance}

Following the usual procedure, a safety report for a new reactorbased neutron source at the Technical University, Munich, was placed before the public for two months at the end of last year. Some 80 people examined it closely, 350 made written objections, and a list containing the names of 50000 people protesting general aspects was submitted. The promoters ot the project (TU Munich and Siemens) will now reply to the issues raised in time for a general discussion in the spring. The Bavarian government will then check in detail every aspect of the design before building permission is sought. Funding is agreed for a final cost below 500 MDM (a useful flux of slightly more than twice that of Orsay's Orphée 14 MW reactor is proposed). The design is based on using highly enriched uranium (instead of low enriched) to ensure a high power density with a high flux of cold neutrons (the most valuable for scientific work). Some argue that although several research reactors use highly enriched fuel, the proposal runs against current regulatory efforts.

Also in Germany, the Steering Committee of the KFA Julich's $23 \mathrm{MW}$ refurbished research reactor has said that it hopes the Land government will give this year the long-awaited approval to restart otherwise it may cease its efforts. Yet another safety evaluation was sought last year in view of a perceived earthquake risk.

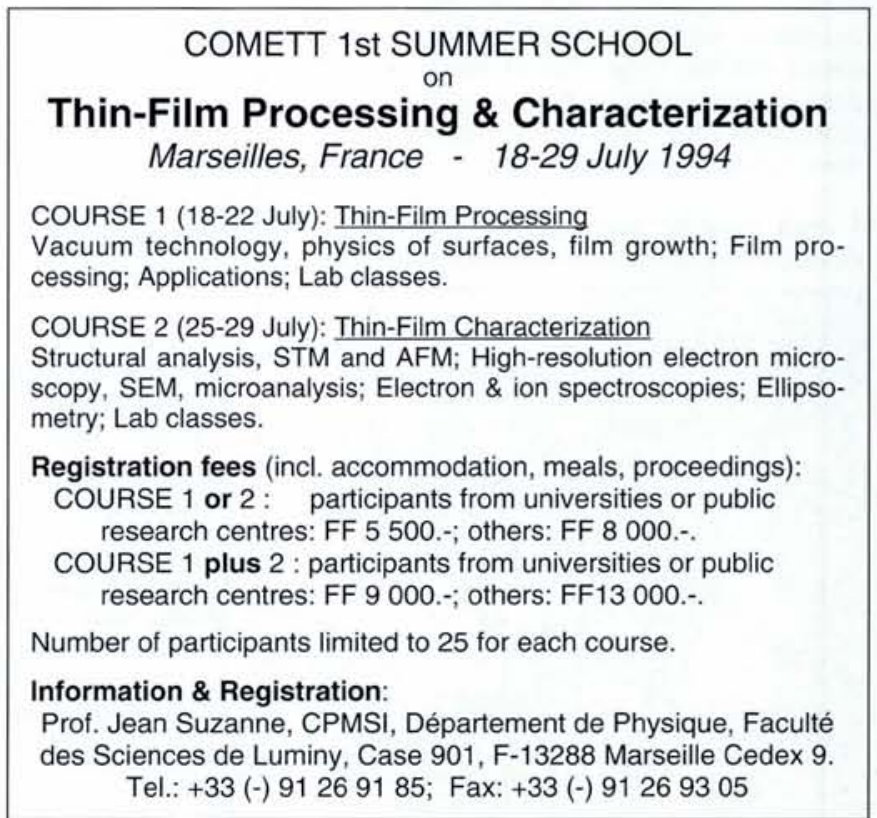

